\title{
A STRUCTURE SHEAF FOR A NONCOMMUTATIVE NOETHERIAN RING
}

\author{
BY BETH GOLDSTON AND A. C. MEWBORN \\ Communicated by Barbara L. Osofsky, June 2, 1975
}

Throughout we assume that $R$ is a left noetherian ring, not necessarily commutative. $R$-modules are left modules, $[M]$ denotes the isomorphism class and $E(M)$ the injective hull of a module $M$.

The (left) spectrum of $R$, denoted $\operatorname{Spec} R$, is taken to be the set of isomorphism classes of indecomposable injective modules. We denote by $G(R)$ the directed graph whose set of vertices is the set $\operatorname{Spec} R$ and such that if $\left[V_{1}\right]$, $\left[V_{2}\right] \in \operatorname{Spec} R$ there is a directed edge from $\left[V_{1}\right]$ to $\left[V_{2}\right]$ in $G(R)$ if and only if there exist critical (see [2, p. 9]) submodules $S_{1}$ and $S_{2}$ of $V_{1}$ and $V_{2}$ and a short exact sequence $0 \rightarrow S_{1} \rightarrow A \rightarrow S_{2} \rightarrow 0, A \subseteq V_{1}$. For a left ideal $I$ of $R$ define $K(I)$ to be the subset of Spec $R$ consisting of [ $V]$ such that there exists $[W] \in \operatorname{Spec} R$, a nonzero map $\alpha: R / I \rightarrow W$, and a directed path in $G(R)$ from $[W]$ to $[V]$. If there is a nonzero module map from $W$ to $V$ there is a directed path in $G(R)$ from $[W]$ to $[V]$.

Proposition 1. The collection of subsets of Spec $R$ of the form $K(I), I$ a left ideal of $R$, is a basis for the closed sets of a topology on $R$. If $R$ is commutative then $\operatorname{Spec} R$ is homeomorphic to the classical spectrum with its Zariski, or hull-kernel, topology.

For each $x \in \operatorname{Spec} R$ we fix an indecomposable injective module $V_{x}$ such that $\left[V_{x}\right]=x$. For a subset $Y$ of Spec $R$ we define $V_{Y}=\amalg_{y \in Y} V_{y}, E_{Y}=$ $\operatorname{End}_{R}\left(V_{Y}\right)$, and $R_{Y}=\operatorname{End}_{E_{Y}}\left(V_{Y}\right)=\operatorname{Biend}_{R}\left(V_{Y}\right)$. We regard $V_{Y}$ as a right $E_{Y}$-module. We define a presheaf of rings over Spec $R$ by letting, for each open subset $U$, the ring of sections over $U$ be $R_{U}$, with the obvious restriction maps.

THEOREM 2. The above presheaf is a sheaf and for each open subset $U$ of Spec $R, R_{U}$ is naturally identified with the quotient ring of $R$ with respect to the torsion theory determined by the injective module $V_{U}$. In particular, $R_{\mathrm{Spec} R}$ is naturally identified with $R$.

If $R$ is commutative the sheaf constructed above reduces to the usual structure sheaf.

If $M$ is an $R$-module the support of $M$, denoted $\operatorname{Supp} M$, is the set of those $[V] \in \operatorname{Spec} R$ such that $M$ is not $V$-torsion. (For definitions and basic properties on torsion theories, see [6].) When $R$ is commutative, the above definition of the

AMS (MOS) subject classifications (1970). Primary 16A08, 16A46, 16 A52. 
support of $M$ agrees with the usual definition.

PROPOSITION 3. If Supp $M$ is the disjoint union of relatively open sets $X_{1}, X_{2}$, then $M$ has a unique decomposition, $M=M_{1} \oplus M_{2}$, where $X_{i}=$ Supp $M_{i}, i=1,2$. In particular, if Spec $R$ is the disjoint union of open subsets $X_{1}, X_{2}$, then $R=R_{1} \oplus R_{2}$, where each $R_{i}$ is a two-sided ideal and Spec $R$ is naturally homeomorphic to $X_{i}, i=1,2$.

If $M$ is an $R$-module we define a presheaf of modules over Spec $R$ by letting for an open set $U$, the module of sections over $U$ be $M_{U}$, where $M_{U}$ is the quotient module of $M$ with respect to the torsion theory determined by $V_{U}$, with the obvious restriction maps.

THEOREM 4. If each open subset of Spec $R$ is compact, the above presheaf is a sheaf and the construction yields a functor from the category of $R$ modules to the category of sheaves of modules over Spec $R$.

If $R$ is commutative the sheaf constructed above reduces to the usual sheaf.

A ring map $\phi: R \rightarrow S$ between noetherian rings $R$ and $S$ is special if for each indecomposable injective $S$-module $V, E\left[{ }_{R} V\right]$ is the direct sum of pairwise isomorphic indecomposable injective $R$-modules. If $\phi$ is special there is a naturally defined map $\phi^{*}: \operatorname{Spec} S \rightarrow \operatorname{Spec} R$ such that $\phi^{*}:\left[{ }_{S} V\right] \rightarrow\left[{ }_{R} W\right]$ where ${ }_{R} W$ is an indecomposable direct summand of $E\left[{ }_{R} V\right]$. We do not know under what conditions $\phi^{*}$ is continuous; however, $\phi^{*}$ does have a property that is closely related to continuity.

We say that a subset $Y$ of $\operatorname{Spec} R$ is closed under generization if Spec $R \backslash Y$ has the property that it contains the closure of each of its points. So open sets are closed under generization.

THEOREM 5. Assume that $\phi: R \rightarrow S$ is special. If $Y \subseteq \operatorname{Spec} R$ is closed under generization, then $\phi^{*-1}(Y) \subseteq \operatorname{Spec} S$ is closed under generization.

A ring map $\phi: R \rightarrow S$ is an extension if $S$ is generated as left $R$-module by the set $\{c \in S \mid c \phi(r)=\phi(r) c$, all $r \in R\}$.

Proposition 6. If $\phi: R \rightarrow S$ is an epimorphism in the category of rings, then $\phi$ is special. If $\phi$ is an extension, then $\phi$ is special.

Several topologies on $\operatorname{Spec} R$ have been defined in [4], [1], and [5]. None of these agree with the topology defined above. For example, if $R$ is the ring of $n \times n$ upper triangular matrices over a field, then $\operatorname{Spec} R$ has $n$ points. In each of the topologies in the listed references, $\operatorname{Spec} R$ is a discrete space, while our topology is the order topology on a linearly ordered set. Our topology is very like that given in [3] where, as in the other references, the noetherian condition is not assumed. We do not know if the topology defined in [3] agrees with our topology for arbitrary left noetherian rings. 
An expanded version of this paper, with proofs and examples, will be published elsewhere.

\section{REFERENCES}

1. Jonathan S. Golan, Topologies on the torsion-theoretic spectrum of a noncommutative ring, Pacific J. Math. 51 (1974), 439-450.

2. Robert Gordon and J. C. Robson, Krull dimension, Mem. Amer. Math. Soc. No. 133 (1973).

3. John Losse, A. C. Mewborn and G. L. Norwood, The spectrum of a noncommutative ring (to appear).

4. Jean Marot, Faisceau des localisations sur un anneau non nécessairement commutatif, C. R. Acad. Sci. Paris Sér. A-B 271 (1970), A1148-A1151. MR 42 \#4578.

5. Jay Shapiro, $A$ noncommutative analog to prime ideals, $\mathrm{Ph}$. D. Thesis, Rutgers University, 1975.

6. Bo T.Stenström, Rings and modules of quotients, Lecture Notes in Math., no. 237, Springer-Verlag, Berlin, 1971. MR 48 \#4010.

DEPARTMENT OF MATEHMATICS, UNIVERSITY OF NORTH CAROLINA, CHAPEL HILL, NORTH CAROLINA 27514 